\title{
Identifying the ideal tracheostomy site based on patient characteristics during percutaneous dilatational tracheostomy without bronchoscopy
}

\author{
Jiho Park ${ }^{1, *}$, Woosuk Chung ${ }^{1,2, *}$, Seunghyun Song ${ }^{1}$, Yoon-Hee Kim ${ }^{1,2}$, \\ Chae Seong Lim ${ }^{1,2}$, Youngkwon Ko ${ }^{1,2}$, Sangwon Yun ${ }^{1}$, Hyunwoo Park ${ }^{1}$, \\ Sangil Park ${ }^{1}$, and Boohwi Hong ${ }^{1,2}$ \\ Department of Anesthesiology and Pain Medicine, ${ }^{1}$ Chungnam National University Hospital, ${ }^{2}$ Chungnam National \\ University College of Medicine, Daejeon, Korea
}

Background: We previously reported that percutaneous dilatational tracheostomy (PDT) can be safely performed $2 \mathrm{~cm}$ below the cricothyroid membrane without the aid of a bronchoscope. Although our simplified method is convenient and does not require sophisticated equipment, the precise location for tracheostomy cannot be confirmed. Because it is recommended that tracheostomy be performed at the second tracheal ring, we assessed whether patient characteristics could predict the distance between the cricothyroid membrane and the second tracheal ring.

Methods: Data from 490 patients who underwent three-dimensional neck computed tomography from January 2012 to December 2015 were analyzed, and the linear distance from the upper part of the cricoid cartilage (CC) to the lower part of the second tracheal ring (2TR) was measured in the sagittal plane.

Results: The mean CC-to-2TR distance was $25.26 \mathrm{~mm}$ (95\% CI 25.02-25.48 mm). Linear regression analysis showed that the predicted CC-to-2TR distance could be calculated as $-5.73+0.2 \times$ height $(\mathrm{cm})+1.22 \times$ sex (male: 1 , female: 0$)+0.01$ $\times$ age $(\mathrm{yr})-0.03 \times$ weight $(\mathrm{kg})\left(\right.$ adj. $\left.\mathrm{R}^{2}=0.55\right)$.

Conclusions: These results suggest that height and sex should be considered when performing PDT without bronchoscope guidance.

Keywords: Airway management; Cricoid cartilage; Critical care; Regression analysis; Trachea; Tracheostomy.

Corresponding author: Boohwi Hong, M.D., Ph.D.

Department of Anesthesiology and Pain Medicine, Chungnam National University Hospital, 282 Munhwa-ro, Jung-gu, Daejeon 35015, Korea

Tel: 82-42-280-7840, Fax: 82-42-280-7968, Email: koho0127@gmail.com

ORCID: https://orcid.org/0000-0003-2468-9271

*Jiho Park and Woosuk Chung contributed equally to this study.

Received: September 19, 2018. Revised: January 1, 2019 (1st); January 28, 2019 (2nd). Accepted: February 11, 2019.

Korean J Anesthesiol 2019 June 72(3): 233-237

https://doi.org/10.4097/kja.d.18.00276

(c) This is an open-access article distributed under the terms of the Creative Commons Attribution Non-Commercial License (http://creativecommons.org/ licenses/by-nc/4.0/), which permits unrestricted non-commercial use, distribution, and reproduction in any medium, provided the original work is properly cited. 


\section{Introduction}

Although airways can be managed initially by intubation with an endotracheal tube [1], prolonged endotracheal intubation has been associated with serious complications. Tracheostomy is an essential procedure for long-term mechanical ventilation and has many additional advantages, including effective removal of secretions, secure tube positioning, improved oral nutritional intake, and ease of weaning from mechanical ventilation.

Percutaneous dilatational tracheostomy (PDT) is a simpler, safer, and less expensive procedure compared to conventional surgical tracheostomy [2]. Fiber-optic bronchoscopy (FOB) during PDT has been shown to prevent complications such as pneumothorax and subcutaneous emphysema, and to confirm proper endotracheal placement [3]. Bronchoscopy can cause complications including hypercarbia and hypoventilation during manipulation of the bronchoscope. This, in turn, may result in acute elevation of intracranial pressure (ICP), especially in patients with acute brain injury [4]. FOB also requires additional medical personnel and equipment [5]. Studies have therefore assessed the safety of PDT without the aid of a bronchoscope [6,7], with several studies reporting that PDT can be safely performed under ultrasound guidance $[8,9]$. However, as with FOB, not all institutions have sophisticated medical devices such as an ultrasonograph.

We previously reported that PDT was safely performed 2 $\mathrm{cm}$ below the cricothyroid membrane without bronchoscopy in 186 patients by confirming the underlying structures through palpation [10]. Although this simplified method is convenient and does not require sophisticated equipment, its biggest drawback was the inability to confirm the precise location for PDT, which is critical when performing PDT $[11,12]$. Although PDT performed approximately at the level of the 2TR [13], patient characteristics can result in anatomical differences [14]. Thus, we examined the effects of patient characteristics on the distance between the cricoid cartilage and the second tracheal ring.

\section{Materials and Methods}

The protocol for this retrospective study was approved by the Institutional Review Board of Chungnam National University Hospital (2015-09-035-005). This study was also registered by the Clinical Research Information Service (KCT0002737). Data from patients who underwent three-dimensional (3D) computed tomography (CT) from January 1, 2012, to December 31, 2015, were retrieved from the Electronic Database of Chungnam National University Hospital. Patients aged > 18 years with physical status of I-III according to the American Society of Anesthesiologists classification system were eligible for inclusion. Patients were excluded if they had a history of neck surgery, chemotherapy, radiation therapy, or any procedure that may have caused an anatomical variation. The 3D neck CT images were analyzed using the picture archiving and communication system (PACS; Maroview, Marosis, Korea) software, and the linear distance from the upper part of the cricoid cartilage (CC) to the lower part of the second tracheal ring (2TR) was measured along the sagittal plane in the 3D CT images (Fig. 1).

\section{Statistical analysis}

Statistical analysis was performed using the R software version 3.5.1 (R Project for Statistical Computing, Austria) and web-based R version 3.5 (web-r.org). One-sample t-test was performed to compare our previous position $(2 \mathrm{~cm}$ below the cricothyroid membrane) with the measured CC-to-2TR distances. The correlations between the CC-to-2TR distance and patient characteristics (sex, height, weight, and age) were assessed by linear regression analysis. Multicollinearity between variables, which can be assessed with the variation inflation factor (VIF), is considered problematic if $\sqrt{\mathrm{VIF}}$ is above 2. Following univariate analysis, variables with $\mathrm{P}$ value $<0.2$ were selected for multivariate backward stepwise regression. Akaike's An Information Criterion (AIC) was used for final model selection. To determine the difference between the model and the actual data, we also calculated the mean absolute percentage error (MAPE) and the root mean squared error (RMSE).

$$
\text { MAPE }=\frac{100}{\mathrm{n}} \sum_{\mathrm{t}=1}^{\mathrm{n}}\left|\frac{\mathrm{A}_{\mathrm{t}}-\mathrm{F}_{\mathrm{t}}}{\mathrm{A}_{\mathrm{t}}}\right| \quad \mathrm{RMSE}=\sqrt{\frac{1}{n} \sum_{t=1}^{n}\left(\mathrm{~A}_{\mathrm{t}}-\mathrm{F}_{\mathrm{t}}\right)^{2}}
$$

The reliability of the predictive model was internally validated by 10 -fold cross-validation. This analysis was performed automatically by the Caret package in R [15]. Four hundred and

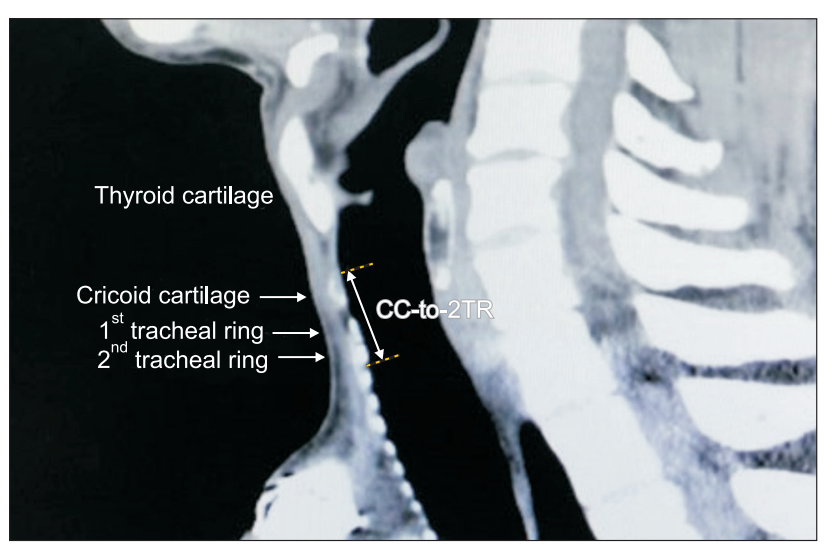

Fig. 1. Representative 3D computed tomography (CT) image. CCto-2TR distance (linear distance from the upper part of the cricoid cartilage to the lower part of the second tracheal ring). 
ninety cases were randomly divided into 10 subsamples (1 test set, 9 training sets). The test group data was examined based on the estimated regression equation developed from the data in the training sets (multiple linear regression [by linear model function in R]). This procedure was repeated until all groups had been tested. The RMSE of 10-fold cross-validation was automatically calculated and may vary from trial to trial, since the 10 subsamples were changed for each trial.

\section{Results}

Of the 496 patients who underwent neck CT, six were excluded due to cervical vertebral fractures. The characteristics of the included patients and their measured CC-to-2TR distances are shown in Table 1. The mean CC-to-2TR distance was 25.2 $\mathrm{mm}$ (95\% CI 25.0-25.5 mm, compared with $2 \mathrm{~cm}, \mathrm{P}<0.001)$. The correlations between CC-to-2TR distance and patient sex, height, weight, and age were determined by simple regression analysis (Fig. 2). All variables were selected for multivariate backward stepwise regression analysis and there was no problem with multicollinearity. The results of the multivariate linear regression analysis for the CC-to-2TR distance are displayed in Table 2. The relative importance of the included factors was $54.5 \%$ for height, $37.3 \%$ for sex, $6 \%$ for weight, and $2.2 \%$ for age (Fig. 3). The final selected model:

CC-to-2TR distance $(\mathrm{mm})=-5.73+0.2 \times$ height $(\mathrm{cm})+1.22$

Table 1. Patient Characteristics and Measured CC-to-2TR Distance

\begin{tabular}{lc}
\hline \multicolumn{1}{c}{ Characteristics } & Value \\
\hline Age $(\mathrm{yr})$ & $48.2 \pm 14.0$ \\
Sex $(\mathrm{F} / \mathrm{M})$ & $319(65) / 171(35)$ \\
Height $(\mathrm{cm})$ & $162.2 \pm 8.5$ \\
Weight $(\mathrm{kg})$ & $63.7 \pm 12.4$ \\
BMI $\left(\mathrm{kg} / \mathrm{m}^{2}\right)$ & $24.1 \pm 4.0$ \\
CC-to-2TR distance $(\mathrm{mm})$ & $25.2 \pm 2.5$ \\
\hline
\end{tabular}

Values are presented as mean \pm SD or number (\%). BMI: body mass index, CC-to-2TR distance: linear distance from the upper part of the cricoid cartilage to the lower part of the second tracheal ring.
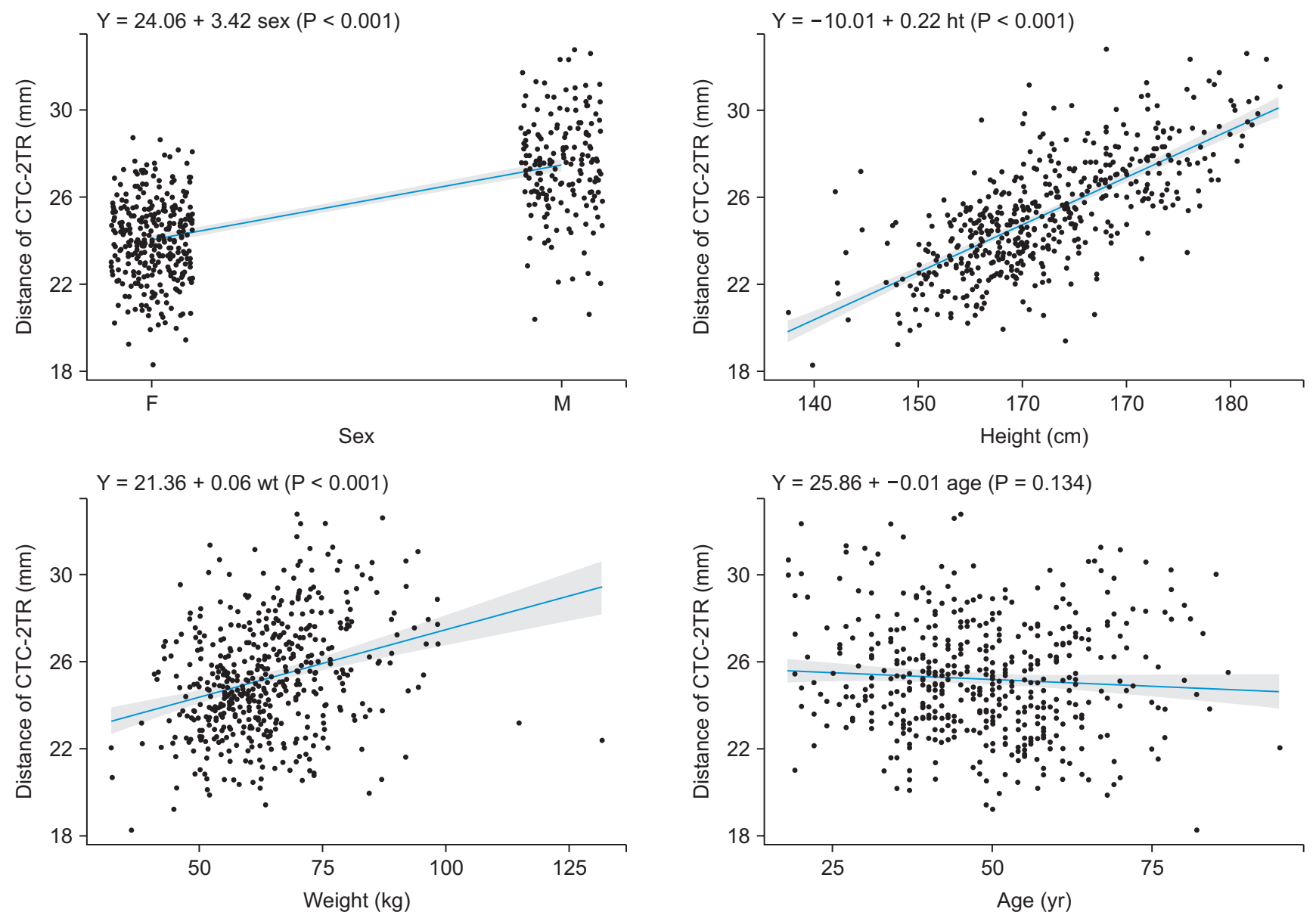

Fig. 2. Simple regression analysis showing factors (sex, height, weight, and age) significantly related to CC-to-2TR distance (linear distance from the upper part of the cricoid cartilage to the lower part of the second tracheal ring). 
Table 2. Multivariate Linear Regression Analysis for CC-to-2TR Distance

\begin{tabular}{lcccr}
\hline \multicolumn{1}{c}{ Variable } & Coefficient & $95 \%$ CI & SE & P value \\
\hline Age (yr) & 0.01 & 0.00 to 0.02 & 0.01 & 0.098 \\
Sex (male) & 1.22 & 0.70 to 1.73 & 0.26 & $<0.001$ \\
Height $(\mathrm{cm})$ & 0.2 & 0.16 to 0.23 & 0.02 & $<0.001$ \\
Weight $(\mathrm{kg})$ & -0.03 & -0.04 to -0.01 & 0.01 & $<0.001$ \\
\hline
\end{tabular}

CC-to-2TR distance: linear distance from the upper part of the cricoid cartilage to the lower part of the second tracheal ring, SE: standard error of the coefficient.

$\times$ sex (male: 1 , female: 0$)+0.01 \times$ age $(\mathrm{yr})-0.03 \times$ weight $(\mathrm{kg})$. (adjusted $\mathrm{R}^{2}=0.55, \mathrm{P}<0.001$ )

The in-sample RMSE was $1.72(\mathrm{~mm})$, indicating that the difference between the actual distance and the estimated distance of the model was $1.72(\mathrm{~mm})$. The MAPE was $5.8 \%$. This indicates that the predicted value of the model may vary by $5.8 \%$. The RMSE of the 10-fold cross validation was $1.73(\mathrm{~mm})$.

\section{Discussion}

Despite tracheostomy being a common procedure in modern medicine, the ideal location to performe tracheostomy has not yet been determined. Both open tracheostomy and PDT are usually performed between the sternal notch and the thyroid cartilage, with the latter being performed approximately at the level of the 2TR [13]. Tracheostomy between the CC and the first tracheal ring is avoided because it may damage the CC, the only circular cartilage of the upper airways. Tracheostomy under the third tracheal ring is also avoided due to the possible presence of the brachiocephalic artery.

Precise positioning has been shown to be important when performing PDT $[11,12]$. The risk of tracheal cartilage fracture, a unique complication that may occur during PDT when the Ciaglia method is used, is also increased in the upper level of the trachea. Due to the backwards angulation of the trachea at its entrance to the thorax, additional force must be applied to dilate the intercartilage space at the upper level near the CC. Because the CC is denser than other types of cartilage, the force is transmitted to the lower cartilage, increasing the risk of cartilage fracture. PDT at lower levels reduces the angle between the dilator and trachea, so that less force is needed [16].

The purpose of this study was to determine the relationship between patient characteristics and the CC-to-2TR distance. Confirmation of these relationships may further enhance the safety of performing PDT in the absence of ultrasound or bronchoscopy guidance. Although previous study have examined the relationships between patient demographics and tracheal anatomy (e.g., length, AP diameter, transverse diameter, and cross-sectional area) [17], no study to date has focused on the

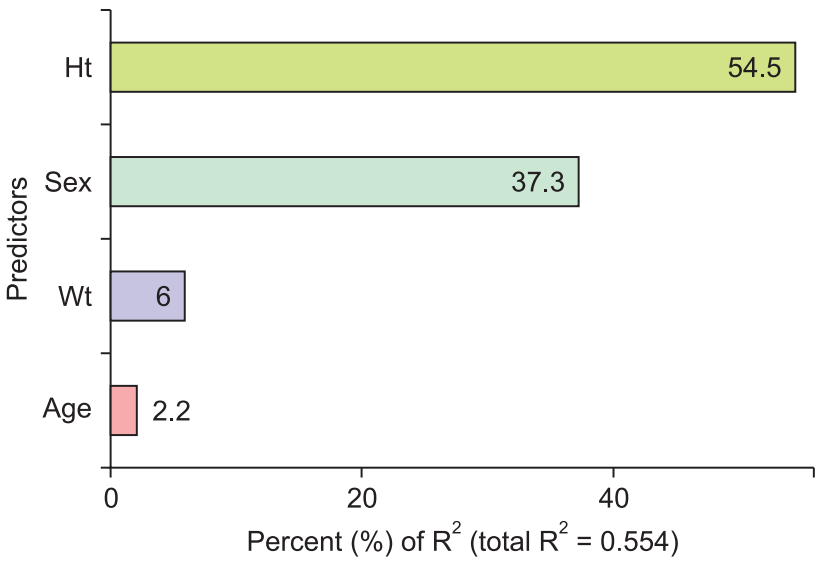

Fig. 3. Relative importance of patient factors on CC-to-2TR distance (linear distance from the upper part of the cricoid cartilage to the lower part of the second tracheal ring). Ht: height $(\mathrm{cm})$, Wt: weight $(\mathrm{kg})$.

distance between the cricothyroid membrane and the tracheal rings. CC-to-2TR distances in our study were measured on $3 \mathrm{D}$ CT images. Unlike bronchoscopy and autopsy, CT does not affect anatomy and is therefore ideal for measurement [18]. Our results suggest that patient characteristics are correlated with the CC-to-2TR distance.

The present study has several limitations. First, our model needs to be externally validated to determine the accuracy of the predictive model. Our regression equation was tested only by internal validation using RMSE and cross validation. Further studies using sophisticated equipment such as an ultrasound may provide additional support for our prediction model. Another important limitation is the difference in patient positioning during tracheostomy and CT scan (tracheostomy, neck extended position; CT scan, neutral neck position). This difference may alter the CC-to-2TR distance. However, Wong et al. [19] reported that the tracheal elongation from a neutral to extended neck position was $1.01 \pm 0.57 \mathrm{~cm}$ when measured with a bronchoscope. Considering the distance from the vocal cords to the carina, which is $13.48 \pm 1.48 \mathrm{~cm}$ in the neutral position, this is only $7 \%$ of the whole tracheal length. The variation due to patient positioning may be insignificant. The third limitation was the homogeneity of the patient population, in that, all patients were Korean. Because the characteristics of patients in western societies differ from those in Korea, our results may not be applicable to other populations.

In conclusion, our results suggest that patient characteristics should be considered when performing PDT without bronchoscopic guidance. The CC-to-2TR distance depends on patient characteristics, especially height and sex. The use of our model may enhance patient safety when performing PDT without the guidance of sophisticated equipment. 


\section{Conflicts of Interest}

No potential conflict of interest relevant to this article was reported.

\section{Author Contributions}

Jiho Park (Writing - review \& editing)

Woosuk Chung (Project administration)

Seunghyun Song (Software)

Yoon-Hee Kim (Writing - review \& editing)

Chae Seong Lim (Writing - review \& editing)

Youngkwon Ko (Resources)

Sangwon Yun (Software)

Hyunwoo Park (Investigation)

Sangil Park (Formal analysis)
Boohwi Hong (Writing - original draft; Writing - review \& editing)

\section{ORCID}

Jiho Park, https://orcid.org/0000-0001-5233-430X

Woosuk Chung, https://orcid.org/0000-0002-6409-2325

Seunghyun Song, https://orcid.org/0000-0001-6674-9645

Yoon-Hee Kim, https://orcid.org/0000-0002-8282-610X

Chae Seong Lim, https://orcid.org/0000-0002-2356-8999

Youngkwon Ko, https://orcid.org/0000-0002-0178-6346

Sangwon Yun, https://orcid.org/0000-0001-8233-4167

Hyunwoo Park, https://orcid.org/0000-0002-1113-4181

Sangil Park, https://orcid.org/0000-0002-2026-6848

Boohwi Hong, https://orcid.org/0000-0003-2468-9271

\section{References}

1. Natanson C, Shelhamer JH, Parrillo JE. Intubation of the trachea in the critical care setting. JAMA 1985; 253: 1160-5.

2. Ciaglia P, Firsching R, Syniec C. Elective percutaneous dilatational tracheostomy. A new simple bedside procedure; preliminary report. Chest 1985; 87: 715-9.

3. Marelli D, Paul A, Manolidis S, Walsh G, Odim JN, Burdon TA, et al. Endoscopic guided percutaneous tracheostomy: early results of a consecutive trial. J Trauma 1990; 30: 433-5.

4. Reilly PM, Anderson HL 3rd, Sing RF, Schwab CW, Bartlett RH. Occult hypercarbia. An unrecognized phenomenon during percutaneous endoscopic tracheostomy. Chest 1995; 107: 1760-3.

5. Jackson LS, Davis JW, Kaups KL, Sue LP, Wolfe MM, Bilello JF, et al. Percutaneous tracheostomy: to bronch or not to bronch--that is the question. J Trauma 2011; 71: 1553-6.

6. Rezende-Neto JB, Oliveira AJ, Neto MP, Botoni FA, Rizoli SB. A technical modification for percutaneous tracheostomy: prospective case series study on one hundred patients. World J Emerg Surg 2011; 6: 35.

7. Agarwal A, Singh D. Is fibreoptic percutaneous tracheostomy in ICU a breakthrough. J Anaesthesiol Clin Pharmacol 2010; 26 : 514-6.

8. Rudas M, Seppelt I, Herkes R, Hislop R, Rajbhandari D, Weisbrodt L. Traditional landmark versus ultrasound guided tracheal puncture during percutaneous dilatational tracheostomy in adult intensive care patients: a randomised controlled trial. Crit Care 2014; 18 : 514.

9. Rajajee V, Fletcher JJ, Rochlen LR, Jacobs TL. Real-time ultrasound-guided percutaneous dilatational tracheostomy: a feasibility study. Crit Care 2011; 15: R67.

10. Chung W, Kim BM, Park SI. Simply modified percutaneous tracheostomy using the Cook ${ }^{\circledR}$ Ciaglia Blue Rhino ${ }^{\mathrm{TM}}$ : a case series. Korean J Anesthesiol 2016; 69: 301-4.

11. Grant CA, Dempsey G, Harrison J, Jones T. Tracheo-innominate artery fistula after percutaneous tracheostomy: three case reports and a clinical review. Br J Anaesth 2006; 96: 127-31.

12. Dollner R, Verch M, Schweiger P, Deluigi C, Graf B, Wallner F. Laryngotracheoscopic findings in long-term follow-up after Griggs tracheostomy. Chest 2002; 122: 206-12.

13. Engels PT, Bagshaw SM, Meier M, Brindley PG. Tracheostomy: from insertion to decannulation. Can J Surg 2009; 52: 427-33.

14. Pak HJ, Hong BH, Lee WH. Assessment of airway length of Korean adults and children for otolaryngology and ophthalmic surgery using a fiberoptic bronchoscope. Korean J Anesthesiol 2010; 59: 249-55.

15. Kuhn M. Building predictive models in R using the caret package. J Stat Softw 2008; 28: 1-26.

16. Karvandian K, Mahmoodpoor A, Mohammadi M, Beigmohammadi M, Jafarzadeh A. Tracheal cartilage fracture with the percutaneous dilatational tracheostomy, Ciaglia method. Middle East J Anaesthesiol 2009; 20: 307-8.

17. Randestad A, Lindholm CE, Fabian P. Dimensions of the cricoid cartilage and the trachea. Laryngoscope 2000; 110: $1957-61$.

18. Griscom NT. Computed tomographic determination of tracheal dimensions in children and adolescents. Radiology 1982; 145: 361-4.

19. Wong DT, Weng H, Lam E, Song HB, Liu J. Lengthening of the trachea during neck extension: which part of the trachea is stretched? Anesth Analg 2008; 107: 989-93. 